\title{
Why do disk galaxies present a common gas-phase metallicity gradient?
}

\section{R. Chang, Shuhui Zhang, Shiyin Shen, Jun Yin and Jinliang Hou}

Key Laboratory for Research in Galaxies and Cosmology, Shanghai Astronomical Observatory, CAS, 80 Nandan Road, Shanghai, 200030, China

email: crx@shao.ac.cn

CALIFA data show that isolated disk galaxies present a common gas-phase metallicity gradient, with a characteristic slope of $-0.1 \mathrm{dex} / r_{e}$ between 0.3 and 2 disk effective radius $r_{e}$ (Sanchez et al. 2014). Here we construct a simple model to investigate which processes regulate the formation and evolution.

Similar to our previous models (Chang et al. 2012), here we also adopt a Gaussian formula of the gas infall rate $f_{\text {in }}(t)=\frac{A}{\sqrt{2 \pi} \sigma} e^{-\left(t-t_{\mathrm{p}}\right)^{2} / 2 \sigma^{2}}$, where the infall-peak time $t_{\mathrm{p}}$ is a free parameter, $A$ is a normalized constant and we fixed $\sigma=3 G y r$. We adopt the classical Schmidt star formation (SF) law as $\Psi=\nu \Sigma_{\text {gas }}^{n}$.

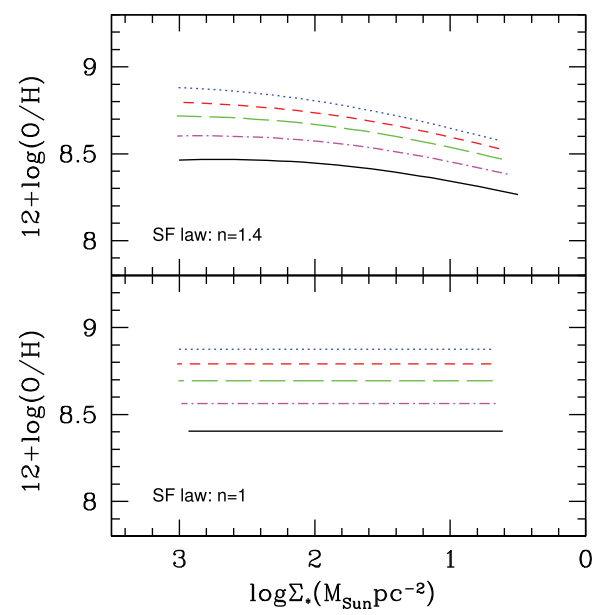

Figure 1. Model results of the gas-phase metallicity versus the stellar mass surface density. Different lines correspond to different gas-infall peak-time $t_{p}$. The upper and lower panel show the results of SF law adopting the power index as $n=1.4$ and $n=1$, respectively. In each panel, the curves represent the results with $t_{\mathrm{p}}=0,3,5,7,9 \mathrm{Gyr}$ from top to bottom.

Fig. 1 shows that, for given $t_{\mathrm{p}}$, the SF law power index $n$ is the main progenitor of radial gradients. Especially, when $n=1$ is adopted, there is no radial gradient. Meantime, for given $n$, if $t_{\mathrm{p}}$ increases with radius, an significant gradient also appears. In other words, both the no-linear SF law and the disk inside-out formation scenario are main progenitors of metallicity gradients and further investigations are needed to explore their degeneracy.

\section{References}

Chang, R. X., Shen, S. Y., \& Hou, J. L. 2012, ApJL, 753, L10

Sanchez, S. F., Rosales-Ortega, F. F., Iglesias-Paramo, J., et al. 2014, A\&\&A, 563, 49 